\title{
Critical appraisal of the use of regorafenib in the management of colorectal cancer
}

This article was published in the following Dove Press journal:

Cancer Management and Research

II April 2013

Number of times this article has been viewed

\author{
Lucia Festino* \\ Alessio Fabozzi* \\ Anna Manzo \\ Valentina Gambardella \\ Erika Martinelli \\ Teresa Troiani \\ Ferdinando De Vita \\ Michele Orditura \\ Fortunato Ciardiello \\ Floriana Morgillo \\ Division of Medical Oncology, \\ Department of clinical and \\ experimental medicine and surgery \\ "F. Magrassi e A. Lanzara", Second \\ University of Naples, Napoli, Italy \\ *These authors contributed equally \\ to this work
}

Correspondence: Floriana Morgillo Assistant Professor, Oncologia Medica, Dipartimento medico chirurgico di internistica clinica e sperimentale "F. Magrassi e A. Lanzara", Seconda Università degli studi di Napoli, II Policlinico, Via Pansini 5, 80I3I Napoli, Italy Tel +3908I 5666725

Fax +3908I 5666732

Email florianamorgillo@yahoo.com

\begin{abstract}
The lack of valid clinical management options for patients affected by metastatic colorectal cancer, which has progressed after all approved standard treatments, has lead to research into new active molecules. Regorafenib is an oral small-molecule multi kinase inhibitor, binding to several intracellular kinases, with powerful inhibitory activity against vascular endothelial growth factor receptors (VEGFR-1,VEGFR-2, and VEGFR-3), platelet-derived growth factor receptor, fibroblast growth factor receptor 1, Raf, TIE-2, and the kinases KIT, RET, and BRAF. The antitumor activity of regorafenib has been tested in vitro and in vivo, and inhibition of tumor growth has been observed in several cancer models, particularly colorectal cancer and gastrointestinal stromal tumors. The most frequent adverse events of grade 3 or higher related to regorafenib were hand-foot skin reaction, fatigue, diarrhea, hypertension, and rash or desquamation. Only a few Phase I-II trials, and most recently a Phase III trial in pretreated colorectal cancer, have been carried out to date. Several ongoing trials are testing the efficacy of regorafenib in combination with chemotherapy. At this point in time, regorafenib is the first small-molecule tyrosine kinase inhibitor to gain approval by the US Food and Drug Administration for pretreated metastatic colorectal cancer patients.
\end{abstract}

Keywords: colorectal cancer, angiogenesis, regorafenib

\section{Introduction to colorectal cancer and management issues}

Colorectal cancer (CRC) is the third most common cancer and the second leading cause of cancer death worldwide. ${ }^{1}$ More than $30 \%$ of patients have locally advanced or metastatic disease at the time of diagnosis, which essentially precludes a cure by surgical treatment alone. ${ }^{2}$ Furthermore, even among patients who undergo apparently curative resection, many experience relapse of CRC, requiring systemic therapy. Standard treatment consists of chemotherapy based on fluoropyrimidines, oxaliplatin, and irinotecan (used in combination or sequentially). However, in the last 20 years an improvement in the outcome of patients with metastatic colorectal cancer (mCRC) has been observed. This is mainly due to the identification of new molecular targets typically altered in CRC cells. ${ }^{3}$ In this context, agents targeting epidermal growth factor receptor (EGFR) and vascular endothelial growth factor (VEGF) and their correlated pathways represent the most promising drugs for the treatment of mCRC. Three of these, cetuximab and panitumumab, monoclonal antibodies against the EGFR, and bevacizumab, a monoclonal antibody against the VEGF protein, have already shown clinical benefit and are commercially approved for use in advanced CRC. ${ }^{46}$ Limitations to the use of anti-EGFR agents have been imposed by the identification of predictive 
biomarkers and molecular mechanisms responsible for intrinsic resistance, such as mutations in the KRAS gene, occurring in $40 \%$ of CRC patients, or BRAF mutations, which occur with a frequency of $5 \%-12 \%$, leading to constitutive activation of the mitogen activated protein kinase (MAPK) pathway independent of the EGFR status. ${ }^{7,8}$ The development of antiangiogenic agents, their introduction into clinical practice, and results from ongoing clinical trials have led to improvements in progression-free survival (PFS) and overall survival (OS) of $\mathrm{mCRC}$ patients. Angiogenesis is the process of new blood vessel formation from a pre-existing vascular bed, essential for tumor growth and metastasis, with a positive correlation between tumor angiogenesis and CRC stage revealed by several studies. ${ }^{9}$

Activation of multiple signaling pathways in the tumor microenvironment, including vascular endothelial growth factor receptors (VEGFRs), fibroblast growth factor receptor (FGFR), and platelet-derived growth factor receptor (PDGFR), controls the initiation of tumor neoangiogenesis. ${ }^{10}$ VEGF was the first vascular-specific growth factor to be characterized, and is one of the most critical drivers of tumor angiogenesis. ${ }^{11}$ VEGF-A is the most important member of the VEGF family (which is comprised of six growth factors: VEGF-A, B, C, D, E, and the placental growth factor), and acts through three VEGF receptors (VEGFR-1 [Flt-1], VEGFR-2 [Flk/KDR], and VEGFR-3 [Flt-4]). ${ }^{12}$ Of the three closely related members of the VEGFR family, the major effects of VEGF on vessel growth and permeability are mediated via VEGFR-2.13,14 Many other growth factors and receptors have been shown to work in a coordinated and complementary manner with established pathways to regulate tumor growth and angiogenesis. This suggests that blockade of multiple growth factor and receptor pathways may be needed to increase the efficacy of cancer therapy. These pathways include FGFR, which is essential for tumor cell proliferation and differentiation via several downstream signaling pathways, ${ }^{15}$ and PDGFR, which modulates the recruitment and maturation of pericytes, supporting vessel stabilization. ${ }^{16}$ The angiopoietin receptor TIE-2 is another angiogenesis tyrosine kinase regulator predominantly expressed on endothelial cells, and is indispensable for the maturation of immature vessels. Its ligands (Ang2/VEGF) are frequently upregulated in several human tumor samples. ${ }^{17-19}$ The tyrosine kinase receptors RET and KIT represent other valid targets for cancer therapy. Mutations of the RET gene have been identified as driving oncogenic events in thyroid carcinomas, ${ }^{20,21}$ and oncogenic KIT mutations are well documented in gastrointestinal stromal tumors (GIST). ${ }^{22,23}$
To date, bevacizumab has been the most successful developed agent; however, not all mCRC patients display tumor response, and even after an initial response to bevacizumab, most of them experience progression of disease. Molecular and cellular mechanisms mediating resistance have been extensively studied. ${ }^{24}$ Therefore, improving the response rate (RR) and OS remains an area of active investigation.

A wide range of angiogenesis inhibitors are currently in development, with a focus on targeting VEGFR-2 to treat cancer. Among these, aflibercept, a fully humanized recombinant fusion protein that binds VEGF-A, VEGF-B, and placental growth factor (PGF)-1 and 2 with high affinity, preventing their binding to VEGF receptors, has demonstrated clinical efficacy in a recent Phase III trial (VELOUR) in secondline treatment of patients with $\mathrm{mCRC}$, in combination with chemotherapy (OS hazard ratio [HR]: 0.82, $P=0.0032$ ). ${ }^{25}$ Several other multi target kinase inhibitors are also in development, often failing to demonstrate an OS improvement in Phase III trials. ${ }^{26-28}$ Regorafenib has been the only promising multi tyrosine kinase inhibitor that has been successfully tested in a Phase III clinical study. This novel oral multi kinase inhibitor has a distinct profile targeting angiogenic, stromal, and oncogenic receptor tyrosine kinases (RTKs).

\section{Pharmacology, mode of action, and pharmacokinetics of regorafenib}

Regorafenib is an oral multi kinase inhibitor targeting both tumor cell proliferation/survival pathways (RAF/MEK/ERK) and selected RTKs such as VEGFR-2/3, TIE-2, PDGFR, RET, and c-KIT (Figure 1).

In vitro, it potently inhibits a distinct set of kinases, including the angiogenic and stromal RTKs VEGFR-1-3, TIE-2, FGFR1 , and PDGFR-b, with half maximal inhibitory concentration $\left(\mathrm{IC}_{50}\right)$ values ranging from $4 \mathrm{nM}$ to $311 \mathrm{nM}$, and the oncogenic RTKs KIT and RET, along with the intracellular signaling kinases c-RAF/RAF-1, BRAF, and V600EBRAF, with $\mathrm{IC}_{50}$ values ranging from $1.5 \mathrm{nM}$ to $28 \mathrm{nM}$. Its ability in inhibiting the wild-type and mutant form of BRAF protein has a great therapeutic implication in the management of $\mathrm{mCRC}$ with $\mathrm{BRAF} /$ V600E that fails to respond to cetuximab or EGFR therapy. ${ }^{29}$ Regorafenib has demonstrated antiproliferative activity in vitro in a wide range of vascular (human umbilical vein endothelial cells and human aortic smooth muscle cells (HASMC)) and tumor (GIST, thyroid, lung, colon, breast, and pancreas) cell lines with different oncogenic mutations. Similar effects were observed in vivo, where once-daily treatment with regorafenib at doses as low as $10 \mathrm{mg} / \mathrm{kg}$ demonstrated broad-spectrum antitumor efficacy in preclinical tumor xenograft models in 


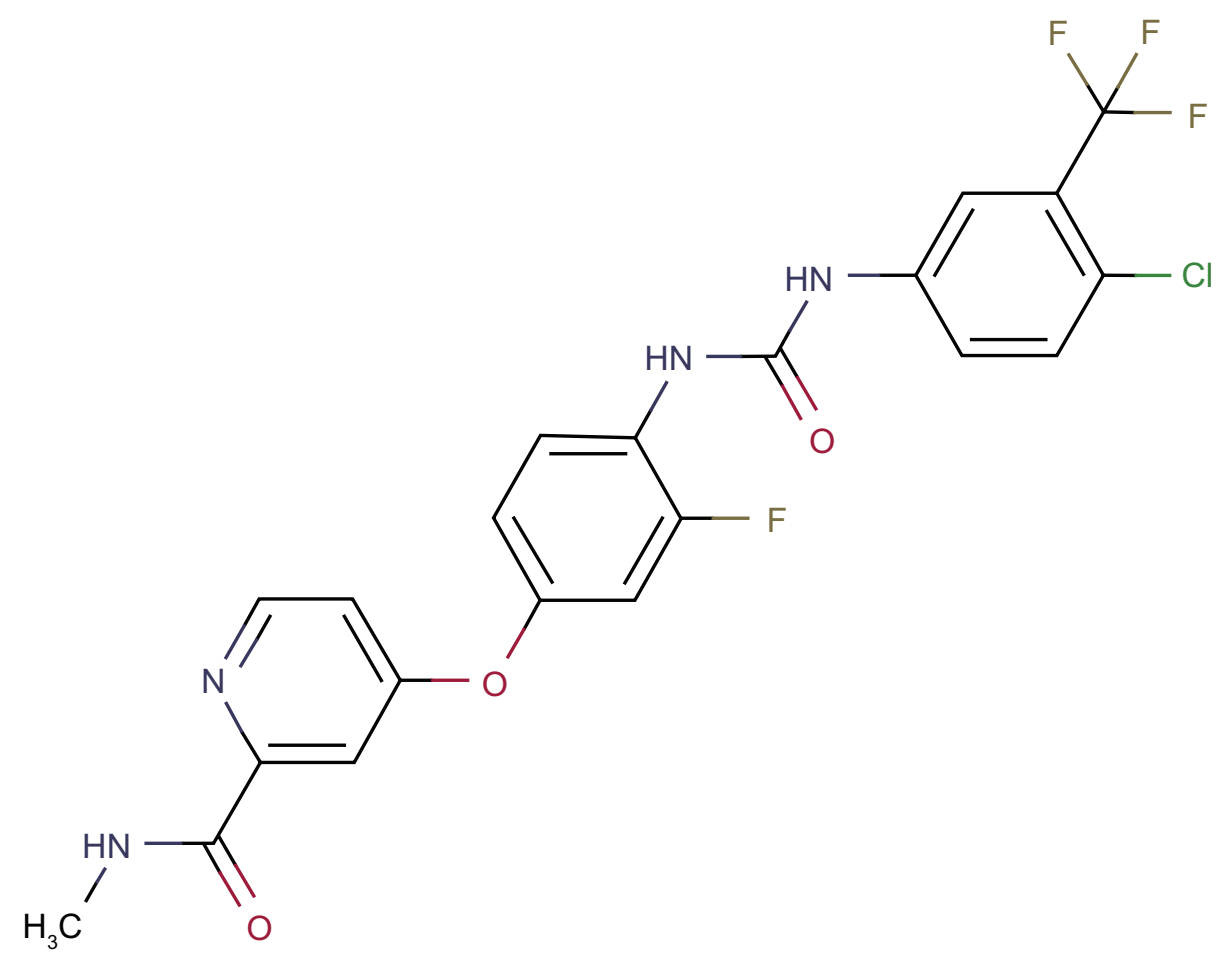

Figure I Regorafenib (BAY 73-4506) molecular structure.

athymic mice (colon [Colo-205(BRAFmt; KRASwt), HT-29 (BRAFmt; KRASwt), HCT-15 (BRAFwt; KRAS mt)], breast, pancreatic, and non-small cell lung cancer). This included the colon HCT-15, a multiple-drug-resistant model, which is resistant to taxanes. Regorafenib effectively inhibited growth of the Colo-205 xenografts in the dose range of $10-100 \mathrm{mg} / \mathrm{kg}$, reaching a tumor growth inhibition of about $75 \%$ at day 14 at the $10 \mathrm{mg} / \mathrm{kg}$ dose. Regorafenib was very well tolerated in mice up to $100 \mathrm{mg} / \mathrm{kg}$ daily $\times 9$ without significant weight loss or animal lethality, suggesting a high apparent therapeutic index.

The antiangiogenic effect of regorafenib was demonstrated in vivo by dynamic contrast-enhanced magnetic resonance imaging. Regorafenib administered once daily orally at $10 \mathrm{mg} / \mathrm{kg}$ significantly decreased the extravasation of Gadomer, a macromolecular magnetic resonance imaging contrast agent suited to display changes in blood vessel permeability, in the vasculature of rat GS9L glioblastoma tumor xenografts. In a daily $(\mathrm{qd}) \times 4$ dosing study, the pharmacodynamic effects persisted for 48 hours after the last dosing and correlated with tumor growth inhibition. A significant reduction in tumor microvessel area was also observed in human colorectal xenografts after treatment with regorafenib. Furthermore, the level of activated pERK1/2, detected by immunohistochemistry, was strongly reduced in treated mice, indicating that, in vivo, regorafenib effectively inhibited the RAF/MEK/ERK signaling cascade.
These data suggest that activity of regorafenib against the tumor proliferation and survival pathway (RAS/RAF/ MAPK) and tumor vasculature pathway represents the basis of its high clinical efficacy.

Pharmacokinetic data were widely studied in preclinical and Phase I clinical trials. Pharmacokinetic analysis revealed a similar exposure at steady state for the parent compound and two pharmacologically active metabolites (M2 and M5). Differences between maximum $\left(\mathrm{C}_{\text {max }}\right)$ and minimum concentration $\left(\mathrm{C}_{\text {min }}\right)$ within the dosing interval of 24 hours were small, with mean $\mathrm{C}_{\max } / \mathrm{C}_{\min }$ ratios of $\sim 2-3$ for both regorafenib and M2, and $\sim 1.5$ for M5. Steady-state pharmacokinetic profiles demonstrated similar mean systemic exposure of regorafenib and its metabolites. Area under the curve (AUC) $)_{0-24}$ and $\mathrm{C}_{\max }$ at steady state showed pronounced variability between patients, with interindividual coefficients of variance of $\sim 60 \%-90 \%$ for regorafenib and M2, and $>100$ for M5. The intraindividual coefficients of variance for $\mathrm{C}_{\max }$ and $\mathrm{AUC}_{0-24}(\mathrm{n}=14)$ were $32 \%-34 \%$ for regorafenib, $37 \%-46 \%$ for $\mathrm{M} 2$, and $52 \%-63 \%$ for M5. Intraindividual variability was therefore markedly reduced compared with interindividual variability. At the $160 \mathrm{mg}$ dose, plasma exposure at steady state $\left(\mathrm{AUC}_{0-24}\right.$, geometric mean) of the metabolites M2 (48 $\left.\mathrm{mg} \mathrm{h} \mathrm{l}^{-1}\right)$ and M5 (65-79 $\mathrm{mg} \mathrm{h}^{-1}$ ) was similar to, or slightly greater than, that of regorafenib (45-50 $\left.\mathrm{mg} \mathrm{h}^{-1}\right)$. The terminal half-life of M2 (25 hours) was comparable with that of regorafenib 
(26-28 hours), although the elimination of M5 was slower, with an estimated half-life of 51-64 hours. ${ }^{30}$

\section{Clinical trials}

Regorafenib has been evaluated as a single agent in two Phase I dose-escalation trials (Table 1). A trial conducted by Mross et $\mathrm{al}^{30}$ recruited 53 patients with solid tumors refractory to standard treatment. The patients were enrolled into eight cohorts at dose levels ranging from $10 \mathrm{mg}$ to $220 \mathrm{mg}$ daily. The recommended dose for future studies was determined to be $160 \mathrm{mg}$ daily, with a treatment schedule of 21 days on/7 days off in repeating 28-day cycles. In this trial, regorafenib showed an acceptable safety profile and preliminary evidence of antitumor activity in patients with solid tumors. Indeed, from a total of 47 patients evaluable for response, three of them achieved a partial response (renal cell carcinoma, CRC, and osteosarcoma). An additional extension cohort was planned to focus on patients with cancer types that were considered to be promising targets for regorafenib. CRC was identified as the tumor type of most interest for further analysis; thus, the extension phase included patients with advanced histologically confirmed CRC.

All the data from the previous Phase I trial were analyzed to study the effects of regorafenib in this setting of patients. Results have been presented for a total of 38 patients with mCRC enrolled during the dose-escalation $(n=15)$ and extension $(n=23)$ phases. The median treatment duration was 53 days. Patients had received extensive previous antitumor treatment including bevacizumab (53\%) and/or anti-

Table I Clinical studies with regorafenib in patients affected by CRC

\begin{tabular}{|c|c|c|c|}
\hline Patients & Treatment & Outcome & References \\
\hline \multicolumn{4}{|l|}{ Phase I } \\
\hline 53 patients & 10-220 mg/daily & 47 patients evaluable & 30 \\
\hline affected by & 21 days on/ & for response: & \\
\hline solid & 7 days off & 3 PR (RCC, CRC, & \\
\hline tumors & every 28 days & osteosarcoma) & \\
\hline 38 CRC & $60-220 \mathrm{mg} /$ daily & & 31 \\
\hline \multirow[t]{3}{*}{ patients } & 21 days on/ & & \\
\hline & 7 days off & & \\
\hline & every 28 days & & \\
\hline \multicolumn{4}{|l|}{ Phase III } \\
\hline 753 CRC & & Median OS & 32 \\
\hline \multicolumn{4}{|l|}{ patients } \\
\hline \multirow[t]{3}{*}{500} & 160 mg/daily & 6.4 months & \\
\hline & 21 days on/ & & \\
\hline & 7 days off & 5.0 months & \\
\hline \multirow[t]{3}{*}{253} & every 28 days & & \\
\hline & Placebo & Hazard ratio 0.77 & \\
\hline & & $\begin{array}{l}95 \% \mathrm{Cl} 0.64-0.94 \\
\text { one-sided } P=0.0052\end{array}$ & \\
\hline
\end{tabular}

Abbreviations: $\mathrm{Cl}$, confidence interval; $\mathrm{CRC}$, colorectal cancer; OS, overall survival; PR, partial response; RCC, renal cell carcinoma; q28, every 28 days.
EGFR antibodies (cetuximab or panitumumab) (53\%). One of 27 evaluable CRC patients achieved a partial response (4\%), and $19(70 \%)$ had stable disease at least 7 weeks after the start of treatment, giving a disease control rate of $74 \%$. Tumor shrinkage was observed in 13 patients. Regorafenib dosing at $160 \mathrm{mg}$ daily using a treatment schedule of 21 days on/7 days off was feasible in patients with advanced refractory CRC. In addition, the study of tumor perfusion in representative lesions measured by dynamic contrast-enhanced magnetic resonance imaging demonstrated decreased tumor vascularization in most patients. ${ }^{31}$

Significant response rates for regorafenib treatment were documented in Phase II clinical studies in patients affected by GIST and non-small cell lung cancer. Consequently, based on the results and the high unmet need in the population of mCRC patients, investigators decided to proceed directly to a Phase III trial.

The CORRECT trial is an international Phase III study conducted to evaluate the efficacy of regorafenib for the treatment of patients with $\mathrm{mCRC}$ after failure of standard therapies (Table 1). The CORRECT trial involved 14 centers in 16 countries in North America, Europe, Asia, and Australia. Between April 2010 and March 2011, 760 patients were randomized to receive regorafenib $(n=505)$ or placebo $(n=255)$. All the patients had histological or cytological documentation of adenocarcinoma of the colon or rectum, had to have received local currently approved standard therapies, and had disease progression during or within 3 months after the last standard therapy. Most baseline characteristics were similar in the regorafenib and placebo groups. However, a lower proportion of patients in the regorafenib group (54\%) had a KRAS mutation compared with the placebo group $(62 \%)$. A similar proportion of BRAF mutations was found in both arms (4\% in placebo group and $6 \%$ in regorafenib group). All patients had been treated with anti-VEGF drugs, although a higher proportion of patients in the placebo group progressed on bevacizumab, irinotecan, and oxaliplatin than in the regorafenib group. The primary endpoint was OS; the secondary efficacy endpoints were PFS, objective RR, and disease control rate and safety. Tumor response and progression were radiologically assessed every 8 weeks. Duration of response, stable disease, and health-related quality of life were assessed as tertiary endpoints. The CORRECT study met its primary endpoint, showing statistically significant improvement in OS of $29 \%(\mathrm{HR}=0.77, P=0.0052)$. Median OS was 6.4 months in the regorafenib group and 5.0 months in the placebo group. Regorafenib showed a benefit in terms of OS in all the subgroups of patients apart from the group of patients with primary disease in the colon and rectum. 
Additionally, findings from the secondary endpoints of the CORRECT study showed statistically significant improvements in PFS $(\mathrm{HR}=0.49, P<0.000001)$; median PFS was 1.9 months in the regorafenib group and 1.7 months in the placebo group. KRAS mutational status was neither prognostic nor predictive in the study population. Both KRAS wild-type and KRAS mutated tumors showed statistically significant improvement in terms of OS (HR 0.653 confidence interval [CI] 0.476-0.895 in KRASwt and HR 0.867 CI 0.670-1.123 in KRASmut) and PFS (HR0.475 CI 0.362-0.623 in KRASwt and HR 0.525 CI 0.425-0.649 in KRASmut) from treatment with regorafenib.

Regorafenib also showed improved disease control rate in patients compared with placebo, although the difference in objective response rate (partial response plus stable disease assessed at least 6 weeks after randomization) between the two arms (1.0\% versus $0.4 \%)$ did not reach statistical significance. No patient had a complete response, but disease control was achieved in $41 \%$ of patients in the regorafenib group and in $15 \%$ in the placebo group. ${ }^{32}$

On the basis of these results, on 27 September 2012 the US Food and Drug Administration approved regorafenib for the treatment of patients with $\mathrm{mCRC}$ who have been previously treated with fluoropyrimidine, oxaliplatin, and irinotecan-based chemotherapy, an anti-VEGF therapy, and, if KRAS wild-type, an anti-EGFR therapy. Expanded access is currently ongoing to provide regorafenib to subjects diagnosed with mCRC who have failed standard therapy and for whom no therapy alternatives exist, in the time between positive results and approval/availability on the market, and to collect safety data for regorafenib until market access.

\section{Ongoing trials}

Several trials evaluating the efficacy of regorafenib in combination with chemotherapy in patients with $\mathrm{mCRC}$ are currently ongoing. The NCT01289821 study is a Phase II trial that will evaluate the efficacy and the safety of regorafenib in combination with chemotherapy (mFOLFOX6) as first-line therapy in patients with mCRC. The primary endpoint of this study is RR; the aim is to show that the therapy of CRC with mFOLFOX6 in combination with regorafenib improves the RR observed for the standard therapy only. Subjects receive regorafenib at a dose of $160 \mathrm{mg}$ daily on days 4-10 and days 18-24 as four $40 \mathrm{mg}$ coprecipitate tablets. Another randomized, multicenter Phase II trial has been designed to compare PFS between regorafenib + FOLFIRI chemotherapy versus placebo + FOLFIRI in patients with $\mathrm{mCRC}$ and KRAS or BRAF mutant disease previously treated with a FOLFOX regimen.

\section{Safety and tolerability profile}

The regorafenib safety profile results were similar to those observed in the drug's Phase I and III trials and typical of the small-molecule tyrosine kinase inhibitor class. They included grade 3 hand-foot skin reactions, fatigue, anorexia, and hypertension controlled with dose reductions. In a Phase I trial conducted by Strumberg et al, ${ }^{31}$ treatment-emergent, drug-related adverse events (AEs) were reported in 32 $(84 \%)$ of the 38 patients with mCRC. All treatment-related AEs were grade 3 or lower, except one case of clinically asymptomatic grade 4 thrombocytopenia. No grade 5 AEs were reported. AEs leading to reduction of the dose were hand-foot skin reactions. Overall, regorafenib was permanently discontinued in eleven patients $(29 \%)$ due to treatment-related AEs. Among the 25 patients treated at the $160 \mathrm{mg}$ dose level, six patients permanently discontinued regorafenib due to treatment-related AEs (hand-foot skin reaction $n=1$, hypertension $n=1$, fatigue $n=1$, fatigue and other constitutional symptoms $\mathrm{n}=1$, thrombocytopenia $\mathrm{n}=1$, duodenal ulcer $\mathrm{n}=1$ ).

A similar safety profile was reported in the Phase III CORRECT trial. Treatment-related AEs (mainly fatigue and hand-foot skin reaction) occurred in $93 \%$ of patients receiving regorafenib compared with $61 \%$ of patients assigned to receive placebo. The most frequent AEs of any grade in the regorafenib group were fatigue and hand-foot skin reactions, and in the placebo group were fatigue and anorexia. Most of them occurred early in the course of treatment (during cycles 1-2). Grade 3-4 treatment-related AEs occurred in $270(54 \%)$ patients assigned to the regorafenib arm. The most frequent regorafenib-related AEs of grade 3 or higher were hand-foot skin reactions, fatigue, diarrhea, hypertension, and rash or desquamation. Serious AEs were reported in $219(44 \%)$ of 500 patients in the regorafenib group and $100(40 \%)$ of 253 patients in the placebo group. Of the 110 deaths reported during the study, only eleven (regorafenib $\mathrm{n}=8,2 \%$; placebo $\mathrm{n}=3,1 \%$ ) were attributed to AEs not associated with disease progression. In the regorafenib group, these AEs were pneumonia $(\mathrm{n}=2)$, gastrointestinal bleeding $(n=2)$, intestinal obstruction $(n=1)$, pulmonary hemorrhage $(n=1)$, seizure $(n=1)$, and sudden death $(n=1)$. Occurrence of thromboembolism did not differ between groups (12 [2\%] patients assigned to regorafenib; four [2\%] patients assigned to placebo). Occurrence of hepatotoxicity was higher in the regorafenib group than in the placebo group. Only one case of death due to regorafenib-related drug-induced liver injury was reported. The most frequent AEs necessitating dose modifications (occurring in 67\% of 500 patients in the regorafenib arm) were dermatological, 
gastrointestinal, constitutional, metabolic, or laboratory events. $^{32}$

\section{Quality of life}

Treatment with regorafenib was not associated with detrimental effects on quality of life, but nor was any improvement shown. ${ }^{33}$ In particular, a careful analysis was carried out by the CORRECT trial. Patients' health-related quality of life and health utility values were measured with the European Organization for Research and Treatment of Cancer Quality of Life Questionnaire (EORTC QLQ-C30) and EuroQol (EQ-5D), respectively (in the EORTC QLQ-C30, the possible score could range from 0 to 100 , with higher scores representing a higher level of functioning and better health-related quality of life; in the EQ-5D, high scores represent better health status). Mean EORTC QLQ-C30 scores at baseline were 62.6 (standard deviation [SD] 21.7) in the regorafenib group and 64.7 (SD 22.4) in the placebo group. Mean scores at the end of treatment were 48.9 (SD 21.6) in the regorafenib group and 51.9 (SD 23.9) in the placebo group. Mean EQ-5D index scores were 0.73 (SD 0.25 ) in the regorafenib group and 0.74 (SD 0.27) in the placebo group at baseline, and 0.59 (SD 0.31 for regorafenib, SD 0.34 for placebo) in each group at the end of the treatment. The mean EQ-5D visual analog scale scores were 65.4 (SD 19.6) in the regorafenib group and 65.8 (SD 20.5) in the placebo group at baseline, and 55.5 (SD 20.4) and 57.3 (SD 21.6), respectively, at the end of treatment. These results suggest that deterioration in patients' quality of life and health status was the same in both the regorafenib and placebo groups. ${ }^{32}$

\section{Discussion}

Innovations in the management of $\mathrm{mCRC}$, with the use of new chemotherapeutic agents and targeted therapies, have doubled median OS from 12 months to almost 24 months. It is now clear that several different subtypes of CRC exist, defined by the acquisition of specific different genetic abnormalities that drive the cell transformation. However, currently, there are limited treatment options in the thirdline setting for patients with $\mathrm{mCRC}$, as a large proportion of patients exhaust all treatment options in earlier lines of therapy. This is particularly relevant for patients with mutations in the KRAS gene who are nonresponsive to anti-EGFR treatments, which are commonly used agents in later lines of therapy. The results of the CORRECT trial confirm that regorafenib is associated with significant improvements in OS, the trial's primary endpoint, PFS, and disease control rate. Based on these results, regorafenib is the first smallmolecule tyrosine kinase inhibitor with approval by the US
Food and Drug Administration in CRC. It is an unexpected triumph against the backdrop of high-profile failures of small-molecule tyrosine kinases in CRC that failed in Phase III first-line studies. Treatment with regorafenib improved significantly OS in advanced colorectal cancer patients $(\mathrm{HR}=0.77)$, indicating a reduction of probability to die of cancer of about $23 \%$, and $51 \%$ of patients treated with regorafenib did not progress. The benefit provided was evident in all the subgroups of patients, including those patients with tumors harboring mutations in the KRAS gene, providing a further tool in the management of this group of patients. Furthermore, regorafenib was demonstrated to be active in a group of patients already pretreated with bevacizumab (although patients were not required to be bevacizumab-refractory). However, although the treatment was not associated with detrimental effects on quality of life, treatment-related toxic events (mainly fatigue and hand-foot skin reaction) occurred in $93 \%$ of patients receiving regorafenib compared with $61 \%$ of patients assigned to receive placebo, and $54 \%$ of patients in the regorafenib group had grade 3 or 4 toxic effects (versus 14\% with placebo).

The results from the CORRECT trial are opening new avenues in the scenario of the management of mCRC. In light of the reported toxic effects, it is necessary, at this point, to identify subgroups of patients, based on molecular markers, who may experience significantly better survival times on regorafenib and are likely to benefit from it the most. In such patients, the OS benefit might be not only statistically significant but also clinically compelling and sufficiently persuasive to justify routine use of regorafenib given the reported side effects.

\section{Disclosure}

The authors have no conflicts of interest to declare.

\section{References}

1. Ferlay J, Shin H-R, Bray F, Forman D, Mathers C, Parkin DM. Estimates of worldwide burden of cancer in 2008: GLOBOCAN 2008. Int J Cancer. 2008;127:2893-2917.

2. Segal NH, Saltz LB. Evolving treatment of advanced colon cancer. Annu Rev Med. 2009;60:207-219.

3. Cohen SJ, Cohen RB, Meropol NJ. Targeting signal transduction pathways in colorectal cancer - more than skin deep. J Clin Oncol. 2005;23:5374-5385.

4. Cunningham D, Humblet Y, Siena S, et al. Cetuximab monotherapy and cetuximab plus irinotecan in irinotecan-refractory metastatic colorectal cancer. $N$ Engl J Med. 2004;351:337-345.

5. Hurwitz H, Fehrenbacher L, Novotny W, et al. Bevacizumab plus irinotecan, fluorouracil, and leucovorin for metastatic colorectal cancer. N Engl J Med. 2004;350:2335-2342.

6. Van Cutsem E, Peeters M, Siena S, et al. Open-label phase III trial of panitumumab plus best supportive care compared with best supportive care alone in patients with chemotherapy-refractory metastatic colorectal cancer. J Clin Oncol. 2007;25(13):1658-1664. 
7. Davies H, Bignell GR, Cox C, et al. Mutations of the BRAF gene in human cancer. Nature. 2002;417(6892):949-954.

8. Andreyev HJN, Norman AR, Cunningham D, et al. Kirsten ras mutations in patients with colorectal cancer: the "RASCAL II" study. $\mathrm{Br} J$ Cancer. 2001;85(5):692-696.

9. Takahashi Y, Kitadai Y, Bucana CD, et al. Expression of vascular endothelial growth factor and its receptor, KDR, correlates with vascularity, metastasis, and proliferation of human colon cancer. Cancer Res. 1995;55:3964-3968.

10. Carmeliet P, Jain RK. Angiogenesis in cancer and other diseases. Nature. 2000;407:249-257.

11. Ferrara N. VEGF and the quest for tumor angiogenesis factor. Nat Rev Cancer. 2002;2(10):795-803.

12. Ellis LM, Hicklin DJ. VEGF-targeted therapy: mechanisms of antitumor activity. Nat Rev Cancer. 2008;8:579-591.

13. Fong GH, Rossant J, Gertsenstein M, Breitman ML. Role of the Flt-1 receptor tyrosine kinase in regulating the assembly of vascular endothelium. Nature. 1995;376:66-70.

14. Shalaby F, Rossant J, Yamaguchi TP, et al. Failure of blood-island formation and vasculogenesis in Flk-1-deficient mice. Nature. 1995; 376:62-66.

15. Acevedo VD, Ittmann M, Spencer DM. Paths of FGFR-driven tumorigenesis. Cell Cycle. 2009;8:580-588.

16. Bergers G, Song S, Meyer-Morse N, Bergsland E, Hanahan D. Benefits of targeting both pericytes and endothelial cells in the tumor vasculature with kinase inhibitors. J Clin Invest. 2003;111:1287-1295.

17. Bach F, Uddin FJ, Burke D. Angiopoietins in malignancy. Eur J Surg Oncol. 2007;33:7-15.

18. Saito M, Watanabe J, Fujisawa T, et al. Angiopoietin-1, 2 and Tie2 expressions in endometrial adenocarcinoma - the Ang2 dominant balance up-regulates tumor angiogenesis in the presence of VEGF. Eur $J$ Gynaecol Oncol. 2006;27:129-134.

19. Szarvas T, Jager T, Totsch M, et al. Angiogenic switch of angiopoietinsTie2 system and its prognostic value in bladder cancer. Clin Cancer Res. 2008; 14:8253-8262.

20. Lanzi C, Cassinelli G, Nicolini V, Zunino F. Targeting RET for thyroid cancer therapy. Biochem Pharmacol. 2009;77:297-309.

21. Nikiforov YE. Thyroid carcinoma:molecular pathways and therapeutic targets Mod Pathol. 2008;21 Suppl 2:37-43.

22. Fletcher JA, Rubin BP. KIT mutations in GIST. Curr Opin Genet Dev. 2007;17:3-7.

23. Heinrich MC, Blanke CD, Drucker BJ, Corless CL. Inhibition of KIT tyrosine kinase activity: a novel molecular approach to the treatmen of KIT-positive malignancies. J Clin Oncol. 2002;20(6):1692-1703.
24. Bergers G, Hanahan D. Modes of resistance to anti-angiogenic therapy. Nat Rev Cancer. 2008;8:592-603.

25. Van Cutsem E, Tabernero J, Lakomy R, et al. Intravenous aflibercept versus placebo in combination with irinotecan/5-FU (FOLFIRI) for second-line treatment of metastatic colorectal cancer (MCRC): results of a multinational phase III trial (EFC10262-VELOUR). Ann Oncol. 2011;22:22.

26. Hecht JR, Trarbach T, Jaeger E, et al. A randomized, double-blind, placebo-controlled, phase III study in patients (Pts) with metastatic a line chemotherapy with oxaliplatin/5-fluorouracil/leucovorin and PTK787/ ZK222584 or placebo (CONFIRM-1). J Clin Oncol. 2005;Suppl 23: Abstract 3.

27. Mross K, Buchert M, Fasol U, et al. A preliminary report of a phase II study of folinic acid, 5-fluorouracil, irinotecan (FOLFIRI) plus sunitinib with toxicity, efficacy, pharmacokinetics, biomarker, imaging data in patients with colorectal cancer with liver metastases as 1st line treatmenta study of the CESAR Central European Society for Anticancer Drug Research - EWIV. Int J Clin Pharmacol Ther. 2011;1:96-98.

28. Siu LL, Shapiro J, Jonker J et al. Phase III randomized trial of cetuximab (CET) plus either brivanib alaninate (BRIV) or placebo in patients (pts) with metastatic (MET) chemotherapy refractory K-RAS wildtype (WT) colorectal carcinoma (CRC): the NCIC Clinical Trials Group and AGITG CO.20 trial. J Clin Oncol. 2012;30(Suppl 4):386.

29. Scott M, Wilhelm SM, Dumas J, et al. Regorafenib (BAY 73-4506): a new oral multikinase inhibitor of angiogenic, stromal and oncogenic receptor tyrosine kinases with potent preclinical antitumor activity. Int J Cancer. 2011;129:245-255.

30. Mross K, Frost A, Steinbild S, Hedbom S, et al. A phase I doseescalation study of regorafenib (BAY 73-4506), an inhibitor of oncogenic, angiogenic, and stromal kinases, in patients with advanced solid tumors. Clin Cancer Res. 2012;18(9):2658-2667.

31. Strumberg D, Scheulen ME, Schultheis B, et al. Regorafenib (BAY 73-4506) in advanced colorectal cancer: a phase I study. Br J Cancer. 2012; 106(11):1722-1777.

32. Grothey A, Van Cutsem E, Sobrero A, et al. Regorafenib monotherapy for previously treated metastatic colorectal cancer (CORRECT): an international, multicentre, randomised, placebo-controlled, phase 3 trial. The Lancet. 2013; 381(9863):303-312.

33. Waddell T, Cunningham D. Evaluation of regorafenib in colorectal cancer and GIST. Lancet Oncol. 2013;381(9863):273-275.
Cancer Management and Research

\section{Publish your work in this journal}

Cancer Management and Research is an international, peer-reviewed open access journal focusing on cancer research and the optimal use of preventative and integrated treatment interventions to achieve improved outcomes, enhanced survival and quality of life for the cancer patient The journal welcomes original research, clinical \& epidemiological

\section{Dovepress}

studies, reviews \& evaluations, guidelines, expert opinion \& commentary, case reports \& extended reports. The manuscript management system is completely online and includes a very quick and fair peerreview system, which is all easy to use. Visit http://www.dovepress.com/ testimonials.php to read real quotes from published authors. 